\title{
Ivacaftor-induced sweat chloride reductions correlate with increases in airway surface liquid $\mathrm{pH}$ in cystic fibrosis
}

Mahmoud H. Abou Alaiwa, ${ }^{1}$ Jan L. Launspach, ${ }^{1}$ Brenda Grogan, ${ }^{2}$ Suzanne Carter, ${ }^{2}$ Joseph Zabner, ${ }^{1}$ David A. Stoltz, ${ }^{1,3}$ Pradeep K. Singh, ${ }^{4}$ Edward F. McKone, ${ }^{2}$ and Michael J. Welsh ${ }^{1,3,5}$

'Department of Internal Medicine and Pappajohn Biomedical Institute, Roy J. and Lucille A. Carver College of Medicine, University of lowa, lowa City, lowa, USA. ${ }^{2}$ National Referral Centre for Adult Cystic Fibrosis, St. Vincent's University Hospital and University College Dublin School of Medicine, Dublin, Ireland. ${ }^{3}$ Department of Molecular Physiology and Biophysics, Roy J. and Lucille A. Carver College of Medicine, University of lowa, lowa City, lowa, USA. ${ }^{4}$ Departments of Medicine and Microbiology, University of Washington, Seattle, Washington, USA. ${ }^{5}$ Howard Hughes Medical Institute, University of lowa, lowa City, lowa, USA.

BACKGROUND. Disruption of cystic fibrosis transmembrane conductance regulator (CFTR) anion channel function causes cystic fibrosis (CF), and lung disease produces most of the mortality. Loss of CFTR-mediated $\mathrm{HCO}_{3}{ }^{-}$secretion reduces the $\mathrm{pH}$ of airway surface liquid (ASL) in vitro and in neonatal humans and pigs in vivo. However, we previously found that, in older children and adults, ASL pH does not differ between CF and non-CF. Here, we tested whether the pH of CF ASL increases with time after birth. Finding that it did suggested that adaptations by CF airways increase ASL $\mathrm{pH}$. This conjecture predicted that increasing CFTR activity in CF airways would further increase ASL pH and also that increasing CFTR activity would correlate with increases in ASL pH.

METHODS. To test for longitudinal changes, we measured ASL pH in newborns and then at 3-month intervals. We also studied people with CF (bearing G551D or R117H mutations), in whom we could acutely stimulate CFTR activity with ivacaftor. To gauge changes in CFTR activity, we measured changes in sweat $\mathrm{Cl}^{-}$concentration immediately before and 48 hours after starting ivacaftor.

RESULTS. Compared with that in the newborn period, ASL pH increased by 6 months of age. In people with CF bearing $\mathbf{C 5 5 1 D}$ or $\mathrm{R} 117 \mathrm{H}$ mutations, ivacaftor did not change the average ASL pH; however reductions in sweat $\mathrm{Cl}^{-}$concentration correlated with elevations of $\mathrm{ASL} \mathrm{pH}$. Reductions in sweat $\mathrm{Cl}^{-}$concentration also correlated with improvements in pulmonary function.

CONCLUSIONS. Our results suggest that CFTR-independent mechanisms increase ASL pH in people with CF. We speculate that CF airway disease, which begins soon after birth, is responsible for the adaptation.

FUNDING. Vertex Inc., the NIH (P30DK089507, 1K08HL135433, HL091842, HL136813, K24HL102246), the Cystic Fibrosis Foundation (SINGH17AO and SINGH15RO), and the Burroughs Wellcome Fund.

Conflict of interest: The authors have declared that no conflict of interest exists.

Submitted: April 4, 2018 Accepted: June 26, 2018 Published: August 9, 2018

Reference information: JCI Insight. 2018;3(15):e121468. https://doi.org/10.1172/jci. insight.121468.

\section{Introduction}

Cystic fibrosis $(\mathrm{CF})$ is caused by mutations in the gene encoding $\mathrm{CF}$ transmembrane conductance regulator (CFTR), and lung disease is the major source of morbidity and mortality (1-3). CFTR is an apical membrane anion channel that provides a pathway for secretion of $\mathrm{Cl}^{-}$and $\mathrm{HCO}_{3}^{-}$into the airway surface liquid (ASL) and submucosal gland lumen $(4,5)$.

The loss of CFTR-mediated $\mathrm{HCO}_{3}{ }^{-}$secretion in $\mathrm{CF}$ predicts that the $\mathrm{HCO}_{3}{ }^{-}$concentration and $\mathrm{pH}$ of ASL and submucosal gland secretions will be reduced. Consistent with this prediction, ASL in differentiated cultures of human $\mathrm{CF}$ airway epithelia has a lower $\mathrm{pH}$ than non-CF epithelia in most measurements (6-9). ASL was also abnormally acidic in airway epithelia cultured from CF pigs (7, 10-13), which develop airway disease similar to that in humans $(14,15)$. Submucosal gland secretions from human CF epithelia 
were also abnormally acidic (16). In addition, in vivo and ex vivo studies of newborn CF pigs revealed an abnormally acidic ASL pH $(7,10)$. In CF rats, in vivo ASL pH was abnormally acidic at $1-6$ months after birth (17). The acidic $\mathrm{pH}$ and reduced $\mathrm{HCO}_{3}^{-}$concentration disrupt respiratory host defense by impairing mucociliary transport and antimicrobial activity $(10,11,18-20)$.

In an earlier pilot study, we measured the $\mathrm{pH}$ of nasal ASL in neonates identified by newborn screening for $\mathrm{CF}$, but before it was known whether or not they had CF. Compared with non-CF neonates, CF neonates ( $<1$ month old) had a lower ASL pH (21). Yet in older children (median age, 3 years) and adults with $\mathrm{CF}$, we found that ASL $\mathrm{pH}$ did not differ from that of people without CF (21). An earlier study also found no $\mathrm{CF} /$ non-CF difference in older children (median age, 12 years) or adults (22). A recent study of children (median age, 4.2 years) also reported no difference between genotypes (9). However, how ASL pH changes in an individual after the neonatal period is unknown.

Our in vivo results suggest that, in CF, ASL $\mathrm{pH}$ may increase with time to reach the $\mathrm{pH}$ of non-CF ASL. That could occur if CFTR-independent mechanisms increase ASL $\mathrm{pH}$ in CF. From that scenario, we made two predictions. (a) If we could precisely reproduce $\mathrm{CF}$-induced secondary changes in non-CF airways, then CFTR-independent mechanisms occurring in addition to $\mathrm{CFTR} \mathrm{HCO}_{3}^{-}$secretion would further increase ASL pH. How we might do this is unclear. (b) If we could acutely restore CFTR activity to $\mathrm{CF}$ airways, then CFTR activity occurring in addition to CFTR-independent changes would raise $\mathrm{pH}$. We hypothesized that, in adults with CF, increasing CFTR activity would increase ASL pH. We also hypothesized that a greater increase in CFTR activity would produce a greater increase in ASL $\mathrm{pH}$.

To test the second prediction, we studied people with CF who carry either a G551D or R117H mutation and increased CFTR activity with ivacaftor. We had an opportunity to perform this study when ivacaftor was approved in Ireland as a treatment for patients carrying a $G 551 D$ or $R 117 H$ mutation. Ivacaftor immediately increases the open-state probability of CFTR-G551D and CFTR-R117H channels, reduces sweat $\left[\mathrm{Cl}^{-}\right]$, and increases pulmonary function measured as the percentage predicted forced expiratory volume in 1 second $\left(\mathrm{FEV}_{1} \%\right)(23-26)$. In addition, when at least one CFTR allele has a G551D mutation, ivacaftor increases ASL $\mathrm{pH}$ in cultured CF airway epithelia (27) and $\mathrm{pH}$ in the small intestinal lumen of people with CF (28). Our hypothesis was that, as CFTR activity increases, ASL pH would increase. To gauge ivacaftor-induced increases in CFTR activity, we measured the change in sweat $\left[\mathrm{Cl}^{-}\right]$. Changes in sweat $\left[\mathrm{Cl}^{-}\right]$ are currently the most reliable and accessible means of assessing changes in CFTR activity in vivo. We also measured changes in $\mathrm{FEV}_{1} \%$. We studied people immediately before and 48 hours after beginning the drug; ivacaftor reaches steady-state concentrations in 2-3 days (29). This approach minimizes secondary consequences of restoring CFTR function and reduces the influence of changes in disease severity.

\section{Results}

CF ASL pH is abnormally acidic at birth and increases with time. In an earlier pilot study, we measured ASL $\mathrm{pH}$ in neonates who screened as positive for immunoreactive trypsinogen before the sweat $\left[\mathrm{Cl}^{-}\right]$or genetic results were known (21). Thus, we were blinded to genotype. Since then, we have studied an additional 5 neonates. Compared with infants without CF, a combined group of $11 \mathrm{CF}$ neonates (Table 1) had a lower ASL pH (Figure 1A).

To test whether ASL pH increases with time, we studied the babies with CF after the initial analysis. Compared with the initial measurement in newborn babies with CF, by 6 months of age, ASL pH was increased (Figure 1B). All the babies had CF pathogens present in cultures and/or neutrophilic inflammation in bronchoalveolar lavage fluid, consistent with previous reports (30-32).

These data suggest that after the neonatal period, CFTR-independent mechanisms increase ASL $\mathrm{pH}$ in $\mathrm{CF}$ airways. The cause is unknown. We speculate that airway inflammation, infection, and remodeling might be responsible. Consistent with that speculation, cytokines that are elevated in CF airways, such as IL-17, IL-1 $\beta$, IL-4, IL-13, and IFN- $\gamma$, raise ASL pH in non-CF epithelia (33-36), and IL-1 $\beta$ has been reported to increase paracellular permeability (37). Moreover, viral infections, with their associated inflammation, can increase ASL pH in vivo (38). However, there are also reports that ASL $\mathrm{pH}$ is decreased in exhaled breath condensate of people with asthma and CF $(39,40)$. Because ASL pH did not differ among neonates, older children, and adults without CF, developmental changes seem less likely to be responsible (21). However, we cannot exclude the possibility that developmental differences in $\mathrm{CF}$ and non-CF airways are responsible. If ASL $\mathrm{pH}$ increases in CF airways after the neonatal period, then acutely adding CFTR activity in addition to those changes should further increase ASL $\mathrm{pH}$. We tested that prediction using ivacaftor. 
Table 1. Clinical characteristics, throat cultures, and bronchoscopy results of neonates with CF

\begin{tabular}{|c|c|c|c|c|c|}
\hline Genotype & IRT (ng/ml) & $\begin{array}{l}\text { Sweat [Cl-] } \\
(\mathrm{mEq} / \mathrm{l})\end{array}$ & ASL pH & Cultures & BALF \\
\hline F508del/G551D ${ }^{A}$ & 195 & 78 & 6.6 & $\begin{array}{c}3 \text { mo and } 15 \text { mo, Pseudomonas aeruginosa and } \\
\text { Staphylococcus aureus }\end{array}$ & $15 \mathrm{mo}, 20 \%$ PMNs \\
\hline F508del/G542XA & 87 & 97 & 4.8 & 6 mo, Pseudomonas aeruginosa & $12 \mathrm{mo}, 50 \% \mathrm{PMNs}$ \\
\hline F508del/F508del ${ }^{A}$ & 231 & 77 & 4.5 & Neg & $18 \mathrm{mo}, 50 \% \mathrm{PMNs}$ \\
\hline G542X/N1303K ${ }^{A}$ & 166 & 93 & 4.8 & 12 mo, Streptococcus & $12 \mathrm{mo}, 15 \% \mathrm{PMNs}$ \\
\hline F508del/F508delA & 132 & 90 & 5.7 & 3 mo, Haemophilus & Not done \\
\hline F508del/N1303K & 202 & 75 & 5.2 & 3 mo, Haemophilus; 6 mo, Moraxella & $6 \mathrm{mo}, 4 \%$ PMNs \\
\hline F508del/F508del & 163 & 95 & 6.9 & $\begin{array}{c}\text { Birth, } 3 \text { mo, } 6 \text { mo, } 9 \text { mo, } 12 \text { mo, and } 18 \text { mo, } \\
\text { Staphylococcus aureus }\end{array}$ & $9 \mathrm{mo}, 12 \%$ PMNs \\
\hline F508del/F508del & 122 & 106 & 4.6 & Birth and 6 mo, Staphylococcus aureus & $6 \mathrm{mo}, 3 \% \mathrm{PMNs}$ \\
\hline
\end{tabular}

IRT, immunoreactive trypsinogen; PMNs, polymorphonuclear cells; BALF, Bronchoalveolar lavage fluid. ${ }^{A}$ Data for these infants can be found in ref. 21. There were no previous longitudinal data for any of these babies. Data for sweat $\left[\mathrm{Cl}^{-}\right]$are average of 2 measurements.

Increases in $\mathrm{ASL}$ pH and $\mathrm{FEV}_{1} \%$ correlate with reductions in sweat [Cl]. We studied people with $\mathrm{CF}$ who carried a $G 551 D$ or $R 117 \mathrm{H}$ mutation and made measurements before and 48 hours after beginning ivacaftor. We previously reported sweat $\left[\mathrm{Cl}^{-}\right]$and $\mathrm{FEV}_{1} \%$ for people with at least one copy of a $G 551 D$ mutation (41-43). Here, we performed a similar study in people with CF carrying at least one $R 117 H$ mutation. We combined the data for sweat $\left[\mathrm{Cl}^{-}\right]$and $\mathrm{FEV}_{1} \%$ for people with either mutation (Table 2 ). The sweat $\left[\mathrm{Cl}^{-}\right]$ fell, and $\mathrm{FEV}_{1} \%$ increased (Figure 2, $\mathrm{A}$ and $\mathrm{B}$ ). We also measured the $\mathrm{pH}$ of nasal ASL in both groups. In contrast to our prediction, ASL pH did not change significantly (Figure 2C). However, given the variance of ASL pH measurements, we were markedly underpowered to detect a difference of $0.2 \mathrm{pH}$ units or greater.

Ivacaftor will increase CFTR activity to variable extents in different individuals. Therefore, we predicted that a greater change in sweat $\left[\mathrm{Cl}^{-}\right]$would be associated with a greater change in $\mathrm{FEV}_{1} \%$. That was the case (Figure 3A). We also predicted that even though ivacaftor did not significantly increase average ASL $\mathrm{pH}$, there would be a correlation between the magnitude of change in CFTR activity (measured as sweat $\left[\mathrm{Cl}^{-}\right]$) and the change in ASL $\mathrm{pH}$. We found that, when ivacaftor produced a greater reduction in sweat $\left[\mathrm{Cl}^{-}\right]$, there was a greater increase in ASL $\mathrm{pH}$ (Figure 3B). There was also a trend $(P=0.13)$ for a positive correlation between increases in $\mathrm{ASL} \mathrm{pH}$ and $\mathrm{FEV}_{1} \%$ (Figure $3 \mathrm{C}$ ). These results suggest that, as CFTR activity increases in CF airways, ASL $\mathrm{pH}$ increases.

\section{Discussion}

This study had several advantages. First, by studying newborns, we could compare neonates with and without CF before airways developed the marked inflammation and infection that characterize CF. Second, the study population of people with ivacaftor-responsive CFTR mutations allowed us to increase CFTR activity in addition to chronic CF-associated changes in the airways. Third, the design of the study took advantage of the immediate effect of ivacaftor on CFTR (23) and the near steady-state concentration achieved within 2 days of beginning administration (29). That allowed us to make measurements at time 0 and 48 hours and thereby diminish any effect of changes in an individual's disease severity, metabolism, or environmental exposures. That short interval likely also minimizes secondary consequences of partially restoring CFTR activity. The ability to minimize confounding factors may explain why we found a correlation between an individual's reduction in sweat $\left[\mathrm{Cl}^{-}\right]$and an increase in $\mathrm{FEV}_{1} \%$, whereas previous studies had only found a correlation between changes in sweat $\left[\mathrm{Cl}^{-}\right]$and $\mathrm{FEV}_{1} \%$ at the population level $(44,45)$. Fourth, in contrast to comparing groups of people with and without $\mathrm{CF}$, individuals served as their own controls. The chance to study a group of people who initiated treatment at the same time at one location provided a unique opportunity to address this question. Fifth, these were in vivo measurements rather than assays in culture. 

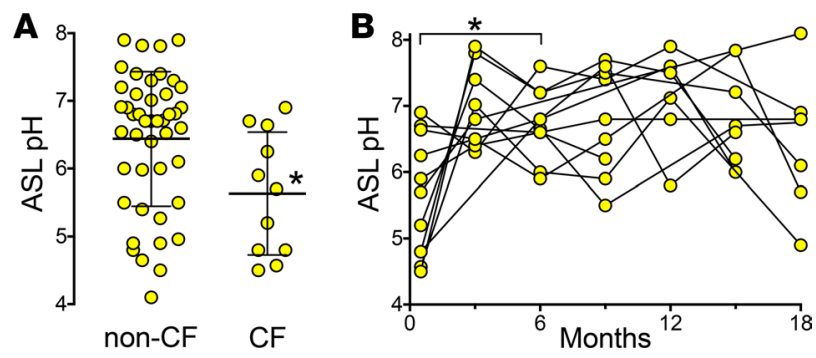

Figure 1. Airway surface liquid pH is abnormally acidic in cystic fibrosis neonates and increases by 3 months of age. (A) The non-CF group includes 4 neonates without cystic fibrosis transmembrane conductance regulator (CFTR) mutations and 41 neonates heterozygous for a CFTR mutation. The cystic fibrosis (CF) group includes 11 neonates. Bars and whiskers indicate mean $\pm \mathrm{SD} .{ }^{*} P=0.01$ by Mann-Whitney test. (B) Airway surface liquid (ASL) $\mathrm{pH}$ in CF neonates $(n=11)$ measured at birth and at 3-month intervals. Data points and connecting lines represent values for individuals. We compared values at birth and at 6 months, a time point when we had data for 10 of the participants. ${ }^{*} P=0.025$ by Wilcoxon matched-pairs signed-rank test.

This study also has limitations. We measured ASL pH on nasal rather than intrapulmonary epithelia. However, the $\mathrm{pH}$ of nasal ASL correlates closely with $\mathrm{pH}$ of lower airway ASL (22). In addition, it would be important to measure $\mathrm{pH}$ in the lumen of submucosal glands where much of the mucus and liquid is generated, but this is not currently possible in vivo $(5,16)$. Another limitation is that ASL $\mathrm{pH}$ values showed substantial variability; how much technical and biological factors contribute to the variation is uncertain.

ASL $\mathrm{pH}$ is controlled by CFTR-mediated $\mathrm{HCO}_{3}^{-}$secretion and by CFTR-independent processes. At birth, loss of CFTR reduces ASL $\mathrm{pH}$. At later time points, CFTR-independent adaptations increase $\mathrm{pH}$ in CF ASL, and CF and non-CF adults and older children have a similar ASL pH, despite the loss of CFTR-mediated $\mathrm{HCO}_{3}^{-}$secretion. Our longitudinal data and the response to ivacaftor support these conclusions but do not identify the non-CFTR mechanisms that increase ASL pH in CF. We speculate that the $\mathrm{pH}$ of non-CF ASL would be higher if non-CF airways had infection, inflammation, and remodeling

Table 2. Baseline clinical characteristics of study subjects

\begin{tabular}{|c|c|c|c|c|}
\hline Genotype & Age & Sex & $\%$ Predicted FEV1 (I) & Sweat $\left[\mathrm{Cl}^{-}\right](\mathrm{mEq} / \mathrm{I})$ \\
\hline G551D/F508del & 23 & $\mathrm{~F}$ & 79 (2.8 L) & 99.9 \\
\hline G551D/F508del & 25 & $\mathrm{~F}$ & $72(2.42 \mathrm{~L})$ & 102.6 \\
\hline G551D/F508del & 23 & $\mathrm{~F}$ & $79(2.46 \mathrm{~L})$ & 90.6 \\
\hline G551D/F508del & 30 & $\mathrm{~F}$ & $40(1.11 \mathrm{~L})$ & 65.7 \\
\hline G551D/F508del & 27 & $\mathrm{~F}$ & 51 (1.49 L) & 91.5 \\
\hline G551D/3659delC & 22 & $\mathrm{~F}$ & 39 (1.25 L) & 109 \\
\hline G551D/F508del & 33 & $\mathrm{~F}$ & $88(2.54 \mathrm{~L})$ & 105 \\
\hline G551D/p67L & 57 & $\mathrm{~F}$ & $34(0.82$ L) & 80.9 \\
\hline G551D/F508del & 35 & $\mathrm{~F}$ & $77(2.16 \mathrm{~L})$ & 98.6 \\
\hline G551D/G551D & 29 & $M$ & 101 (3.99 L) & 110.3 \\
\hline G551D/F508del & 30 & $M$ & $39(1.6 \mathrm{~L})$ & 108.2 \\
\hline G551D/R117H & 33 & $M$ & $72(2.76 \mathrm{~L})$ & 61.9 \\
\hline R117H/F508del & 45 & $M$ & $108(3.80 \mathrm{~L})$ & 98 \\
\hline R117H/F508del & 40 & $M$ & 96 (3.75 L) & 81 \\
\hline R117H/F508del & 41 & $\mathrm{~F}$ & $87(2.44 \mathrm{~L})$ & 61 \\
\hline R117H/F508del & 34 & $\mathrm{~F}$ & $62(1.81 \mathrm{~L})$ & 71 \\
\hline R117H/F508del & 40 & $M$ & 75 (3.05 L) & 78 \\
\hline R117H/F508del & 52 & $M$ & $69(2.39 \mathrm{~L})$ & 90 \\
\hline R117H/M156R & 42 & $\mathrm{~F}$ & 43 (1.37 L) & 61 \\
\hline R117H/M156R & 40 & $M$ & 35 (1.49 L) & 66 \\
\hline $\mathrm{R} 117 \mathrm{H} / 2622+1 \mathrm{G} \rightarrow \mathrm{A}$ & 25 & $M$ & $75(3.41 \mathrm{~L})$ & 89 \\
\hline R117H/F508del & 46 & $\mathrm{~F}$ & $62(1.90 \mathrm{~L})$ & 80 \\
\hline
\end{tabular}



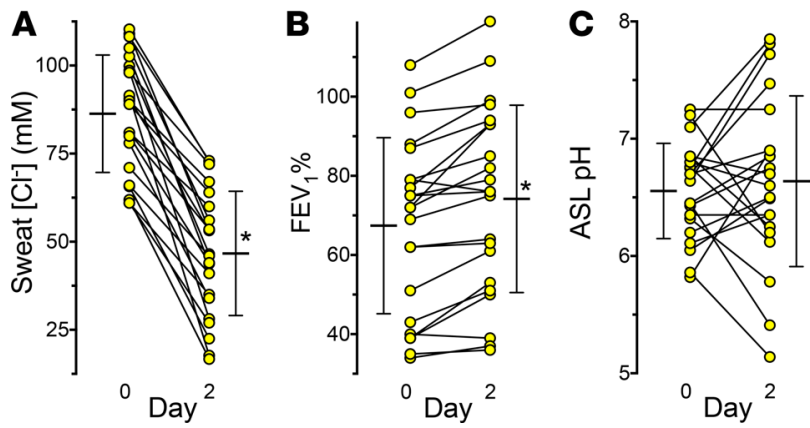

Figure 2. Ivacaftor acutely reduces sweat $\mathrm{Cl}^{-}$concentration and increases forced expiratory volume in 1 second. (A) Sweat $\mathrm{Cl}^{-}$concentration, (B) forced expiratory volume in 1 second ( $\mathrm{FEV} \%$ ), and (C) airway surface liquid (ASL) pH on day 0 and 2 days after beginning ivacaftor. Each pair of data points and connecting lines indicate a different person. $n=22$ for sweat [ $\left.\mathrm{Cl}^{-}\right]$and $\mathrm{FEV}, \% ; n=21$ for ASL pH (1 person declined ASL pH measurements). Bars and whiskers indicate mean $\pm \mathrm{SD}$. We did not detect an effect of sex or genotype on the results. ${ }^{*} P<0.001$ by Wilcoxon matched-pairs signed-rank test.

identical to that in $\mathrm{CF}$ airways. Conversely, we speculate that the $\mathrm{pH}$ of ASL in CF adults might be lower in the absence of CFTR-independent adaptations.

These results may also have implications for respiratory host defense. Previous studies showed that there may not be a single optimal value for ASL $\mathrm{pH}$; as $\mathrm{pH}$ and $\mathrm{HCO}_{3}{ }^{-}$concentration increase, MCT and antimicrobial activity increase $(10-12,46)$. Therefore, increasing ASL $\mathrm{pH}$ may be of therapeutic benefit.

Thus, a better understanding of the mechanisms responsible for

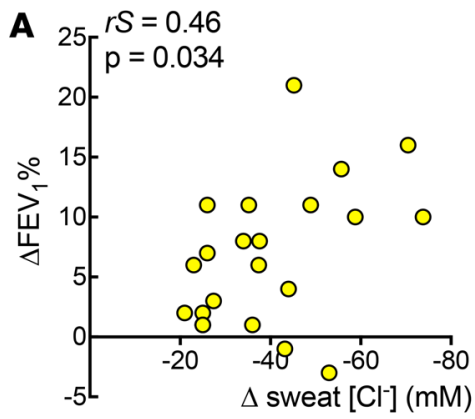
a CFTR-independent increase in ASL $\mathrm{pH}$ might be exploited to enhance airway defense in CF and perhaps other airway diseases.

\section{Methods}

Study participants. From April 2012 until June 2016, in Iowa, we enrolled 56 newborns who had a screening immunoreactive trypsinogen $\geq 65 \mathrm{ng} / \mathrm{ml}$. We measured nasal ASL $\mathrm{pH}$ at their first clinic visit (age varied from a few days after birth to $<1$ month), before results of the sweat $\left[\mathrm{Cl}^{-}\right]$or genetic test results were known. For many of the participants, we were able to repeat the measurement of nasal ASL $\mathrm{pH}$ at 3-month intervals over the

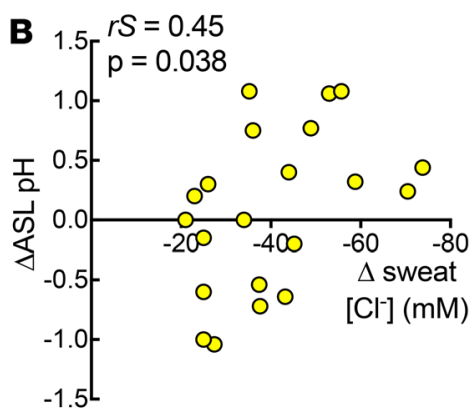
course of 18 months. Table 1 shows the genotypes, sweat $\left[\mathrm{Cl}^{-}\right]$, and initial ASL $\mathrm{pH}$. The table also shows evidence of the development of CF airway disease based on throat swab cultures and fiber optic bronchoscopic examination of the airways with collection of bronchoalveolar lavage.

We enrolled adult subjects with CF who had at least one $G 551 D$ or $R 117 H$ allele in two prospective observational studies at a single CF center, the National Referral Centre for Adult Cystic Fibrosis. Inclusion criteria included an age $\geq 18$ years (both male and female subjects were enrolled), a clinical diagnosis of CF and

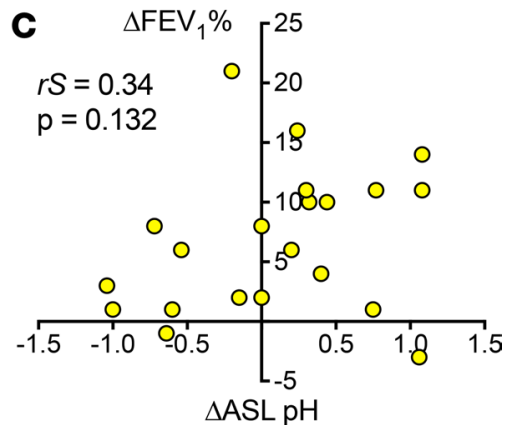

Figure 3. Ivacaftor-induced changes in sweat $\mathrm{Cl}^{-}$concentration correlate with changes in forced expiratory volume in 1 second and airway surface liquid pH. (A) Relationship between changes in sweat $\mathrm{Cl}^{-}$concentration and changes in forced expiratory volume in 1 second $\left(\mathrm{FEV}_{1} \%\right)(P=0.034$ Spearman rank correlation, 0.46 correlation coefficient, $n=22$ ). (B) Relationship between changes in sweat $\mathrm{Cl}^{-}$concentration and changes in airway surface liquid (ASL) pH $(P=0.038$ Spearman rank correlation, 0.45 correlation coefficient, $n=21$ ). (C) Relationship between changes in ASL pH and changes in $\mathrm{FEV}_{1} \%(P=0.132$ Spearman rank correlation, 0.34 correlation coefficient, $n=21$ ). 
a G551D-CFTR or R117H-CFTR mutation on at least 1 allele, clinical stability with no significant changes in health status within 14 days prior to visit 1 , and a negative serum pregnancy test at screening for females of childbearing potential. Exclusion criteria included participation in the VX-770 Extended Access Program or use of ivacaftor within 6 months prior to visit 1; an acute upper or lower respiratory infection, pulmonary exacerbation, or changes in therapy (including antibiotics) for pulmonary disease within 4 weeks of visit 1 ; history of solid-organ transplantation; ongoing pregnancy or breastfeeding; ongoing participation in another therapeutic clinical study or prior participation in an investigational drug study within 30 days before screening; use of any inhibitors or inducers of cytochrome P450 3A4; any non-CF-related illness within 2 weeks before visit 1 ; or abnormal renal or liver function at screening. Standard therapy for $\mathrm{CF}$ was allowed to continue, except for inhaled bronchodilators that were held the morning of visits. Table 2 summarizes the clinical characteristics of participants. Measurements of sweat $\left[\mathrm{Cl}^{-}\right]$, spirometry, and ASL $\mathrm{pH}$ were obtained at baseline and 2 days after starting ivacaftor. Values of sweat $\left[\mathrm{Cl}^{-}\right]$and $\mathrm{FEV}_{1} \%$ for participants with a $G 551 D$ mutation have been reported previously (41-43).

Sweat $\left[\mathrm{Cl}^{-}\right]$. Sweat was collected with the Macroduct collection system (Wescor), and sweat $\left[\mathrm{Cl}^{-}\right]$was measured using routine laboratory techniques.

Spirometry. Spirometric measurements were obtained in accordance with the American Thoracic Society Standards. The FEV ${ }_{1}$ predicted values are based on the European Community for Coal and Steel 1993 values for adults.

$A S L p H$. We used a Sandhill ZepHr PHNS-P (Sandhill Scientific) Mobidium $\mathrm{pH}$ probe with an internal reference electrode to measure ASL $\mathrm{pH}$ in the nose. Prior to each study, the $\mathrm{pH}$ probe was calibrated in buffer solutions of $\mathrm{pH} 6,7$, and 8 (VWR). Voltage was recorded with an Oakton pH6 + meter (Cole-Parmer) and corrected to temperature. The probe was positioned $1 \mathrm{~cm}$ (neonates) or $6 \mathrm{~cm}$ (adults) from the most caudal aspect of the columella. The catheter remained in position until the reading was stable for 15 seconds. All measurements were taken by the same operator. One person in the ivacaftor study declined measurement of ASL $\mathrm{pH}$.

Statistics. Data are presented as values for individual participants with mean \pm SD indicated by bars. For statistical analysis, we used a Mann-Whitney test for unpaired comparisons and reported significance with a 2 -tailed $P$ value. For paired comparisons, we used Wilcoxon matched-pairs signed-rank test and reported significance with a 2-tailed $P$ value. In Figure 1B, at 6 months, we had ASL pH measurements from 10 of the 11 neonates. Therefore, we used this time point to compare to measurements at birth. In Figure 3, we tested correlations with a nonparametric Spearman correlation coefficient. Differences were considered statistically significant at $P<0.05$.

Study approval. All participants (and their guardians, as applicable) provided written informed consent. The studies were approved by the University of Iowa Institutional Review Board and the Research Ethics Committee of St. Vincent's University Hospital.

\section{Author contributions}

MHAA, JZ, DAS, PKS, EFM, and MJW led the study design. MHAA, JLL, BG, SC, JZ, DAS, PKS, EFM, and MJW contributed to data collection and data analyses. MHAA, JZ, DAS, PKS, EFM, and MJW interpreted the data. MHAA and MJW drafted the manuscript. MHAA, JLL, BG, SC, JZ, DAS, PKS, EFM, and MJW contributed to revisions.

\section{Acknowledgments}

We thank the participants who made this study possible. We are grateful to Beth Dowd and Lauri Ramsey for help with study participants. This study was supported by an investigator-initiated award from Vertex Inc. (to PKS and EFM), the Cystic Fibrosis Foundation (SINGH17A0 and SINGH15R0), and the NIH (P30DK089507). MHAA is supported in part by the NIH (1K08HL135433). JZ is supported in part by the NIH (HL091842). DAS is supported in part by the NIH (HL136813). PKS is supported in part by the NIH (K24HL102246) and the Burroughs Wellcome Fund. MJW is an Investigator of the Howard Hughes Medical Institute.

Address correspondence to: Michael J. Welsh, Howard Hughes Medical Institute, Carver College of Medicine, 6332 PBDB, University of Iowa, Iowa City, Iowa 52242, USA. Phone: 319.335.7619; Email: michael-welsh@uiowa.edu. 
1. Quinton PM. Physiological basis of cystic fibrosis: a historical perspective. Physiol Rev. 1999;79(1 Suppl):S3-S22.

2. Stoltz DA, Meyerholz DK, Welsh MJ. Origins of cystic fibrosis lung disease. N Engl J Med. 2015;372(4):351-362.

3. Cutting GR. Cystic fibrosis genetics: from molecular understanding to clinical application. Nat Rev Genet. 2015;16(1):45-56.

4. Poulsen JH, Fischer H, Illek B, Machen TE. Bicarbonate conductance and $\mathrm{pH}$ regulatory capability of cystic fibrosis transmembrane conductance regulator. Proc Natl Acad Sci USA. 1994;91(12):5340-5344.

5. Lee RJ, Limberis MP, Hennessy MF, Wilson JM, Foskett JK. Optical imaging of Ca2+-evoked fluid secretion by murine nasal submucosal gland serous acinar cells. J Physiol (Lond). 2007;582(Pt 3):1099-1124.

6. Coakley RD, et al. Abnormal surface liquid $\mathrm{pH}$ regulation by cultured cystic fibrosis bronchial epithelium. Proc Natl Acad $S c i$ USA. 2003;100(26):16083-16088.

7. Shah VS, et al. Airway acidification initiates host defense abnormalities in cystic fibrosis mice. Science. 2016;351(6272):503-507.

8. Garland AL, et al. Molecular basis for pH-dependent mucosal dehydration in cystic fibrosis airways. Proc Natl Acad Sci USA. 2013;110(40):15973-15978.

9. Schultz A, et al. Airway surface liquid pH is not acidic in children with cystic fibrosis. Nat Commun. 2017;8(1):1409.

10. Pezzulo AA, et al. Reduced airway surface $\mathrm{pH}$ impairs bacterial killing in the porcine cystic fibrosis lung. Nature. 2012;487(7405):109-113.

11. Tang XX, et al. Acidic pH increases airway surface liquid viscosity in cystic fibrosis. J Clin Invest. 2016;126(3):879-891.

12. Shah VS, et al. Relationships among CFTR expression, HCO3- secretion, and host defense may inform gene- and cell-based cystic fibrosis therapies. Proc Natl Acad Sci USA. 2016;113(19):5382-5387.

13. Li X, et al. Electrolyte transport properties in distal small airways from cystic fibrosis pigs with implications for host defense. $A m$ J Physiol Lung Cell Mol Physiol. 2016;310(7):L670-L679.

14. Stoltz DA, et al. Cystic fibrosis pigs develop lung disease and exhibit defective bacterial eradication at birth. Sci Transl Med. 2010;2(29):29ra31.

15. Ostedgaard LS, et al. The $\triangle \mathrm{F} 508$ mutation causes CFTR misprocessing and cystic fibrosis-like disease in pigs. Sci Transl Med. 2011;3(74):74ra24.

16. Song Y, Salinas D, Nielson DW, Verkman AS. Hyperacidity of secreted fluid from submucosal glands in early cystic fibrosis. $A m$ J Physiol, Cell Physiol. 2006;290(3):C741-C749.

17. Birket SE, et al. Development of an airway mucus defect in the cystic fibrosis rat. JCI Insight. 2018;3(1):e97199.

18. Quinton PM. Cystic fibrosis: impaired bicarbonate secretion and mucoviscidosis. Lancet. 2008;372(9636):415-417.

19. Gustafsson JK, et al. Bicarbonate and functional CFTR channel are required for proper mucin secretion and link cystic fibrosis with its mucus phenotype. J Exp Med. 2012;209(7):1263-1272.

20. Hoegger MJ, et al. Impaired mucus detachment disrupts mucociliary transport in a piglet model of cystic fibrosis. Science. 2014;345(6198):818-822.

21. Abou Alaiwa MH, et al. Neonates with cystic fibrosis have a reduced nasal liquid pH; a small pilot study. $J$ Cyst Fibros. 2014;13(4):373-377.

22. McShane D, Davies JC, Davies MG, Bush A, Geddes DM, Alton EW. Airway surface pH in subjects with cystic fibrosis. Eur Respir J. 2003;21(1):37-42.

23. Van Goor F, et al. Rescue of CF airway epithelial cell function in vitro by a CFTR potentiator, VX-770. Proc Natl Acad Sci USA. 2009;106(44):18825-18830.

24. Accurso FJ, et al. Effect of VX-770 in persons with cystic fibrosis and the G551D-CFTR mutation. N Engl J Med. 2010;363(21):1991-2003.

25. Ramsey BW, et al. A CFTR potentiator in patients with cystic fibrosis and the G551D mutation. N Engl J Med. 2011;365(18):1663-1672.

26. Yu H, et al. Ivacaftor potentiation of multiple CFTR channels with gating mutations. J Cyst Fibros. 2012;11(3):237-245.

27. Chang EH, et al. Medical reversal of chronic sinusitis in a cystic fibrosis patient with ivacaftor. Int Forum Allergy Rhinol. 2015;5(2):178-181.

28. Gelfond D, et al. Impact of CFTR modulation on intestinal $\mathrm{pH}$, motility, and clinical outcomes in patients with cystic fibrosis and the G551D mutation. Clin Transl Gastroenterol. 2017;8(3):e81.

29. Vertex P. Ivacaftor prescribing information. Vertex Pharmaceuticals. https://pi.vrtx.com/files/uspi_ivacaftor.pdf. Accessed July 19, 2018.

30. Stick SM, et al. Bronchiectasis in infants and preschool children diagnosed with cystic fibrosis after newborn screening. $J$ Pediatr. 2009;155(5):623-8.e1.

31. Khan TZ, Wagener JS, Bost T, Martinez J, Accurso FJ, Riches DW. Early pulmonary inflammation in infants with cystic fibrosis. Am J Respir Crit Care Med. 1995;151(4):1075-1082.

32. Armstrong DS, et al. Lower airway inflammation in infants and young children with cystic fibrosis. Am J Respir Crit Care Med. 1997;156(4 Pt 1):1197-1204.

33. Gorrieri G, et al. Goblet cell hyperplasia requires high bicarbonate transport to support mucin release. Sci Rep. 2016;6:36016

34. Haggie PM, Phuan PW, Tan JA, Zlock L, Finkbeiner WE, Verkman AS. Inhibitors of pendrin anion exchange identified in a small molecule screen increase airway surface liquid volume in cystic fibrosis. FASEB J. 2016;30(6):2187-2197.

35. Kreindler JL, et al. Interleukin-17A induces bicarbonate secretion in normal human bronchial epithelial cells. Am J Physiol Lung Cell Mol Physiol. 2009;296(2):L257-L266.

36. Gray T, et al. Regulation of MUC5AC mucin secretion and airway surface liquid metabolism by IL-1beta in human bronchial epithelia. Am J Physiol Lung Cell Mol Physiol. 2004;286(2):L320-L330.

37. Coyne CB, Vanhook MK, Gambling TM, Carson JL, Boucher RC, Johnson LG. Regulation of airway tight junctions by proinflammatory cytokines. Mol Biol Cell. 2002;13(9):3218-3234.

38. Fabricant ND. Relation of the $\mathrm{pH}$ of nasal secretions in situ to the activity of lysozyme. Arch Otolaryngol. 1945;41:53-55

39. Carpagnano GE, Barnes PJ, Francis J, Wilson N, Bush A, Kharitonov SA. Breath condensate $\mathrm{pH}$ in children with cystic fibrosis and asthma: a new noninvasive marker of airway inflammation? Chest. 2004;125(6):2005-2010.

40. Hunt JF, et al. Endogenous airway acidification. Implications for asthma pathophysiology. Am J Respir Crit Care Med. 
2000;161(3 Pt 1):694-699.

41. Adam RJ, et al. Acute administration of ivacaftor to people with cystic fibrosis and a G551D-CFTR mutation reveals smooth muscle abnormalities. JCI Insight. 2016;1(4):e86183.

42. Hisert KB, et al. Restoring cystic fibrosis transmembrane conductance regulator function reduces airway bacteria and inflammation in people with cystic fibrosis and chronic lung infections. Am J Respir Crit Care Med. 2017;195(12):1617-1628.

43. Hisert $\mathrm{KB}$, et al. Ivacaftor-induced proteomic changes suggest monocyte defects may contribute to the pathogenesis of cystic fibrosis. Am J Respir Cell Mol Biol. 2016;54(4):594-597.

44. Fidler MC, Beusmans J, Panorchan P, Van Goor F. Correlation of sweat chloride and percent predicted FEV1 in cystic fibrosis patients treated with ivacaftor. J Cyst Fibros. 2017;16(1):41-44.

45. Seliger VI, Rodman D, Van Goor F, Schmelz A, Mueller P. The predictive potential of the sweat chloride test in cystic fibrosis patients with the G551D mutation. J Cyst Fibros. 2013;12(6):706-713.

46. Abou Alaiwa MH, et al. $\mathrm{pH}$ modulates the activity and synergism of the airway surface liquid antimicrobials $\beta$-defensin-3 and LL-37. Proc Natl Acad Sci USA. 2014;111(52):18703-18708. 